\title{
Incoherent excitation of few-level multiatom ensembles
}

\author{
Baranidharan Mohan ${ }^{1}$ and Mihai A. Macovei ${ }^{1,2}$ \\ ${ }^{1}$ Max-Planck-Institut für Kernphysik, Saupfercheckweg 1, D-69117 Heidelberg, \\ Germany \\ ${ }^{2}$ Institute of Applied Physics, Academiei str. 5, MD-2028 Chişinău, Moldova \\ E-mail: ph1100833@physics.iitd.ac.in \\ E-mail: mihai.macovei@mpi-hd.mpg.de
}

\begin{abstract}
We investigate the collective interaction of a multi-atom three-level ladder system with an environmental incoherent reservoir. The exact solution of the master equation that describes such a system of atoms emitting distinguishable or indistinguishable photons on interaction with an incoherent reservoir is obtained. The quantum decay interference effects have no influences over steady-state distribution of population among the energy levels. However, the photon statistics of the collectively emitted photons are different in this case. In particular, the normalized second-order photon correlation function shows a minimum in its value for the photons generated on lower atomic transition in a system with orthogonal dipoles. The minimum is due to the steady-state amplified spontaneous emission by the incoherent applied fields.
\end{abstract}

Submitted to: J. Phys. B: At. Mol. Phys.

PACS numbers: 42.50.Fx, 42.50.Lc, 42.50.Ar 


\section{Introduction}

The collective behavior of quantum systems on interaction with a common radiation field was first studied by Dicke [1]. Since then, the studies regarding the collective interactions of an ensemble of few-level emitters with and via an environmental reservoir and pumped by external coherent or incoherent sources of electromagnetic light have been carried out intensively [1, 2, 3, 4, 5, 6]. The investigation of such interactions has interested the scientific community because of the interesting effects and the possible application in the field of quantum communication and quantum information. The manipulation of collective fluorescence of an atomic sample via classical coherent fields and a heat bath has been shown in Ref. [7]. Further, it has been shown that the photon scattering by a collection of few-level emitters in incoherent environments leads to violation of the Cauchy-Schwarz inequality [8]. Thus, it can be realized that, in contrary to general intuition, quantum features can be obtained from the interaction of quantum systems with a classical electromagnetic field (EMF) reservoir. This opens up a lot of possible applications in quantum information science, for example, entanglement between two arbitrary qubits has been shown to be generated when they interact with a common thermal bath [9, 10, 11, 12. Recently, other interesting developments like, stationary entanglement at high temperatures for two coupled, parametrically driven, dissipative harmonic oscillators [13] and room-temperature steady-state optomechanical entanglement on a chip [14], have been shown. Disentanglement versus decoherence of two qubits in thermal noise was investigated as well [15.

With such a motivation, in this paper, we investigate the quantum behaviors of a collection of three-level ladder emitters surrounded by an incoherent reservoir. We find that the steady-state distribution of radiators on the energy levels are not affected by the presence of the cross-damping terms caused by the interference of transition amplitudes. The collective effects drive the system into a final thermal steady-state which is other than Boltzmann equilibrium distribution. The photon statistics changes from super-Poissonian to sub-Poissonian depending on the number of atoms in the sample, temperature and the mutual orientation of the induced dipole-moments. In particular, we analyzed the steady state intensity and the normalized second-order correlation function for the light generated on the lower atomic transition. We found that there occurs a maximum in the steady-state intensity for moderately large atomic samples with orthogonal transition dipoles. The physics behind such a behavior is that the steady-state intensity of the emitted photons for the lower transition has a maximum due to the amplified spontaneous emission by the incoherent field. Correspondingly, a minimum is observed in the normalized second-order correlation function, resulting in the emission of quasi-coherent light. When the orientation of the transition dipoles are near parallel, the dip is not observed and the photon statistics is similar to that of a two-level ensemble.

The paper is organized as follows. In Section 2, we consider the system of interest and obtain the exact steady-state solution of the master equation that describes the 
system. Using the solution, we arrive at the distribution of the emitters on the atomic states. Section 3 investigates the photon statistics of the spontaneously emitted photons as function of number of atoms, bath characteristics and orientation of atomic dipoles. The results are summarized in Section 4.

\section{Master Equation and its exact steady state solution}

The basic element of our investigation is a sample of $N$ identical non-overlapping threelevel ladder emitters that interacts with an environmental incoherent reservoir like thermal bath or broad-band incoherent lasers. The radiating atoms are located within a volume with linear dimension less compared to the relevant emission wavelengths $\left\{\lambda_{12}, \lambda_{23}\right\}$ (Dicke model). However, the obtained results apply to extended atomic samples as well where one atomic dimension is much larger than the relevant emission wavelength [16. The incoherent reservoir induces transitions between the atomic levels with rates proportional to the mean incoherent photon number at corresponding atomic transitions. The excited atomic level $|1\rangle(|2\rangle)$ spontaneously decays to the state $|2\rangle(|3\rangle)$ due to the zero point fluctuation of the electromagnetic field, with a decay rate $2 \gamma_{1}\left(2 \gamma_{2}\right)$.

In the usual mean field, Born-Markov, dipole and rotating wave approximations, the interaction of the atomic sample with the surrounding incoherent bath is described by the master equation [2]:

$$
\begin{aligned}
& \frac{d}{d t} \rho(t)+i\left[\omega_{12} S_{11}-\omega_{23} S_{33}, \rho\right]=-\left(1+\bar{n}_{1}\right)\left[S_{12},\left(\gamma_{1} S_{21}+\gamma_{21} S_{32}\right) \rho\right] \\
- & \left(1+\bar{n}_{2}\right)\left[S_{23},\left(\gamma_{2} S_{32}+\gamma_{12} S_{21}\right) \rho\right]-\bar{n}_{1}\left[S_{21},\left(\gamma_{1} S_{12}+\gamma_{21} S_{23}\right) \rho\right] \\
- & \bar{n}_{2}\left[S_{32},\left(\gamma_{2} S_{23}+\gamma_{12} S_{12}\right) \rho\right]+H . c .
\end{aligned}
$$

where the collective atomic operators $S_{\alpha \beta}=\Sigma_{j=1}^{N} S_{\alpha \beta}^{(j)}=\Sigma_{j=1}^{N}|\alpha\rangle_{j j}\langle\beta|\equiv| \alpha\rangle\langle\beta|$ describe the transitions between $|\beta\rangle$ and $|\alpha\rangle$ for $\alpha \neq \beta$ and populations for $\alpha=\beta$ and obey the commutation relation $\left[S_{\alpha \beta}, S_{\alpha^{\prime} \beta^{\prime}}\right]=\delta_{\beta \alpha^{\prime}} S_{\alpha \beta^{\prime}}-\delta_{\beta^{\prime} \alpha} S_{\alpha^{\prime} \beta}$. Here, $\bar{n}_{i}$ are the mean photon numbers that represent the intensity of incoherent pumping. For thermal bath, the mean thermal photon number is given by,

$$
\bar{n}_{i}=\frac{1}{\exp \left(\beta \hbar \omega_{i, i+1}\right)-1},
$$

where, $\omega_{i, i+1}=\omega_{i}-\omega_{i+1},\{i=1,2\}$ and $\beta=\left(k_{B} T\right)^{-1}$ where $k_{B}$ is the Boltzmann constant and $\mathrm{T}$ is the temperature of the bath. For incoherent pumping,

$$
\bar{n}_{i}=\frac{R_{i, i+1} d_{i, i+1}^{2}}{\gamma_{i} \hbar^{2}},
$$

where $R_{i, i+1}$ describes the strength of the incoherent pumping. Furthermore, $2 \gamma_{1(2)}$ $=4 d_{12(23)}^{2} \omega_{12(23)}^{3} /\left(3 \hbar c^{3}\right)$ is the single- atom natural line width. $\gamma_{12}=\gamma_{2} \frac{\left|\vec{d}_{12}\right|}{\left|\vec{d}_{23}\right|} \cos \theta$ and $\gamma_{21}=\gamma_{1} \frac{\left|\vec{d}_{23}\right|}{\left|\vec{d}_{12}\right|} \cos \theta$, with $\theta$ being the angle between the dipole moments $\vec{d}_{12}$ and $\vec{d}_{23}$, describe the interference (cross-damping) effects among the atomic transitions $|1\rangle \leftrightarrow|2\rangle$ 
and $|2\rangle \leftrightarrow|3\rangle$, and cannot be neglected for non-orthogonal dipole moments if $\Delta=$ $\left|\omega_{12}-\omega_{23}\right| \leq \Gamma_{\text {eff }}=N \gamma_{1(2)}\left[1+\bar{n}_{1(2)}\right]$.

The steady-state solution of the master equation, Eq. (1) is given by the relation:

$$
\rho_{s}=Z^{-1} e^{-\xi_{1} S_{11}} e^{-\xi_{3} S_{33}},
$$

where for $\gamma_{12}=\gamma_{21}=0$ (i.e. when $\overrightarrow{d_{12}} \perp \overrightarrow{d_{23}}$ ),

$$
\xi_{1}=\ln \left[\frac{1+\bar{n}_{1}}{\bar{n}_{1}}\right], \quad \xi_{3}=\ln \left[\frac{\bar{n}_{2}}{1+\bar{n}_{2}}\right]
$$

while for $\bar{n}_{1}=\bar{n}_{2} \equiv \bar{n}$ and $\gamma_{12}=\gamma_{21} \neq 0$,

$$
\xi_{1}=-\xi_{3} \equiv \xi=\ln \left[\frac{1+\bar{n}}{\bar{n}}\right] .
$$

Here $Z$ is chosen such that $\operatorname{Tr}\left\{\rho_{\mathrm{s}}\right\}=1$. It is interesting to emphasize that the exact solution, i.e. Eq. (2), has a diagonal form while the master equation itself Eq. (1) has non-diagonal terms which arise due to the two transitions $|1\rangle \rightarrow|2\rangle$ and $|2\rangle \rightarrow|3\rangle$ coupling with the same electromagnetic field modes. The solution of the master equation (11) was obtained by the direct substitution of Eq. (2) in the steady-state form of Eq. (1), noting that: $e^{\xi_{1} S_{11}} S_{21} e^{-\xi_{1} S_{11}}=S_{21} e^{-\xi_{1}}$ and $e^{\xi_{3} S_{33}} S_{32} e^{-\xi_{3} S_{33}}=S_{32} e^{\xi_{3}}$.

The steady-state expectation values of the atomic variables of interest can be calculated using the expression for the steady-state solution of the master equation, i.e. Eq. (2), and by making use of the symmetrical collective states $|N, n, m\rangle$ corresponding to the $\mathrm{SU}(3)$ algebra [2, 4, 5, 7, 8]. The meaning of the symmetrical collective state $|N, n, m\rangle$ is such that, $n$ atoms are considered to be in the bare state $|1\rangle, m-n$ in the state $|2\rangle$, and $N-m$ in the bare state $|3\rangle$, where $N \geq n \geq 0, N \geq m \geq n$. Using the $\mathrm{SU}(3)$ eigenstate properties of the bare state atomic operators we can immediately arrive at the expression for $Z$ (see, also [17]), i.e.,

$$
Z\left(\xi_{1}, \xi_{3}\right)=\frac{e^{-\xi_{3} N}}{1-e^{\xi_{3}}}\left[f\left(\xi_{1}-\xi_{3}\right)-e^{\xi_{3}(1+N)} f\left(\xi_{1}\right)\right]
$$

Here,

$$
f(\xi)=\frac{1-e^{-\xi(1+N)}}{1-e^{-\xi}}
$$

Both the collective steady-state populations on the bare atomic states and their mutual correlations can be calculated from the relations:

$$
\left\langle S_{11}^{k_{1}} S_{33}^{k_{2}}\right\rangle_{s}=(-1)^{k_{1}+k_{2}} Z^{-1} \frac{\partial^{k_{1}+k_{2}}}{\partial \xi_{1}^{k_{1}} \partial \xi_{3}^{k_{2}}} Z\left(\xi_{1}, \xi_{3}\right),
$$

with $\left\langle S_{22}\right\rangle_{s}=N-\left\langle S_{11}\right\rangle_{s}-\left\langle S_{33}\right\rangle_{s},\left\{k_{1}, k_{2}=0,1,2 \cdots\right\}$. In particular, for $N=1$ one obtains

$$
\begin{aligned}
\left\langle S_{11}\right\rangle_{s} & =\frac{\bar{n}_{1} \bar{n}_{2}}{\left(1+2 \bar{n}_{1}\right)\left(1+2 \bar{n}_{2}\right)-\bar{n}_{1}\left(1+\bar{n}_{2}\right)}, \\
\left\langle S_{22}\right\rangle_{s} & =\frac{\bar{n}_{2}\left(1+\bar{n}_{1}\right)}{\left(1+2 \bar{n}_{1}\right)\left(1+2 \bar{n}_{2}\right)-\bar{n}_{1}\left(1+\bar{n}_{2}\right)}, \\
\left\langle S_{33}\right\rangle_{s} & =\frac{\left(1+\bar{n}_{1}\right)\left(1+\bar{n}_{2}\right)}{\left(1+2 \bar{n}_{1}\right)\left(1+2 \bar{n}_{2}\right)-\bar{n}_{1}\left(1+\bar{n}_{2}\right)},
\end{aligned}
$$


and it can be observed that their corresponding ratios are in accordance with the equilibrium Boltzmann distribution.

In general, for any $N$, the population distribution of collection of atoms are given by:

$$
\begin{aligned}
& \left\langle S_{11}\right\rangle_{s}=\frac{\eta_{2}^{-N}}{Z\left(\xi_{1}, \xi_{3}\right)\left(\eta_{2}-1\right)}\left[\frac{(N+1)\left(\eta_{1} \eta_{2}\right)^{N+1}-N\left(\eta_{1} \eta_{2}\right)^{N+2}-\eta_{1} \eta_{2}}{\left(1-\eta_{1} \eta_{2}\right)^{2}}\right. \\
- & \left.\eta_{2}^{N+1} \frac{(N+1) \eta_{1}^{N+1}-N \eta_{1}^{N+2}-\eta_{1}}{\left(1-\eta_{1}\right)^{2}}\right], \\
& \left\langle S_{33}\right\rangle_{s}=\frac{\eta_{2}^{-N}}{Z\left(\xi_{1}, \xi_{3}\right)\left(\eta_{2}-1\right)^{2}} \\
\times & {\left[\frac{N+\left(\eta_{1} \eta_{2}\right)^{N+1}-\eta_{2}\left(\eta_{1} \eta_{2}\right)^{N+2}-\eta_{1} \eta_{2}\left(1-(N+2) \eta_{2}+N\right)-(N+1) \eta_{2}}{\left(1-\eta_{1} \eta_{2}\right)^{2}}\right.} \\
+ & \left.\eta_{2}^{N+1} \frac{1-\eta_{1}^{N+1}}{1-\eta_{1}}\right],
\end{aligned}
$$

with $\left\langle S_{22}\right\rangle_{s}=N-\left\langle S_{11}\right\rangle_{s}-\left\langle S_{33}\right\rangle_{s}$ and $\eta_{i}=\bar{n}_{i} /\left[1+\bar{n}_{i}\right],\{i \in 1,2\}$. In this case, for larger $N$, the collective interaction between the atoms drives the system into a thermal steady-state away from a Boltzmann distribution [8].

Now, we consider the following limiting cases of the applied incoherent field and the size of the system:

i) for a system with $\eta_{1}=0, \eta_{2} \neq 0$, we find that,

$$
\begin{aligned}
\left\langle S_{11}\right\rangle_{s} & =0 \\
\left\langle S_{33}\right\rangle_{s} & =\frac{N-(N+1) \eta_{2}+\eta_{2}^{N+1}}{\left(1-\eta_{2}\right)\left(1-\eta_{2}^{N+1}\right)},
\end{aligned}
$$

i.e. we recovered the well-known results for a two-level $(|2\rangle \leftrightarrow|3\rangle)$ atomic sample [3].

ii) if $\left\{\eta_{1} \neq 0, \eta_{2}=0\right\}$ or when there is no external incoherent pumping, $\left\{\eta_{1}=\eta_{2}=\right.$ $0\}$, then $\left\langle S_{11}\right\rangle_{s}=0$ and $\left\langle S_{33}\right\rangle_{s}=N$. The system is entirely in the ground state.

iii) for a weak incoherent bath $\left(\eta_{i}<1\right)$ and a large sample $(N \gg 1)$ such that $\left\{\left(\eta_{1} \eta_{2}\right)^{N}, \eta_{1(2)}^{N}\right\} \rightarrow 0$, we get,

$$
\begin{aligned}
\left\langle S_{11}\right\rangle_{s} & =\frac{\eta_{1} \eta_{2}}{1-\eta_{1} \eta_{2}}, \\
\left\langle S_{22}\right\rangle_{s} & =\frac{\eta_{2}}{1-\eta_{2}}, \\
\left\langle S_{33}\right\rangle_{s} & =N-\frac{\eta_{2}}{1-\eta_{2}}-\frac{\eta_{1} \eta_{2}}{1-\eta_{1} \eta_{2}},
\end{aligned}
$$

iv) on application of a strong incoherent field $\left(\eta_{i}=1\right)$, we get,

$$
\lim _{\eta_{1}, \eta_{2} \rightarrow 1} \frac{\left\langle S_{11}\right\rangle_{s}}{N}=\lim _{\eta_{1}, \eta_{2} \rightarrow 1} \frac{\left\langle S_{22}\right\rangle_{s}}{N}=\lim _{\eta_{1}, \eta_{2} \rightarrow 1} \frac{\left\langle S_{33}\right\rangle_{s}}{N}=\frac{1}{3}
$$

i.e., the atomic levels are equally populated (see Fig. 1).

When cross-damping effects are considered [18] then the corresponding expressions for the population distributions can be obtained with the help of Eq. (2) in the limit 


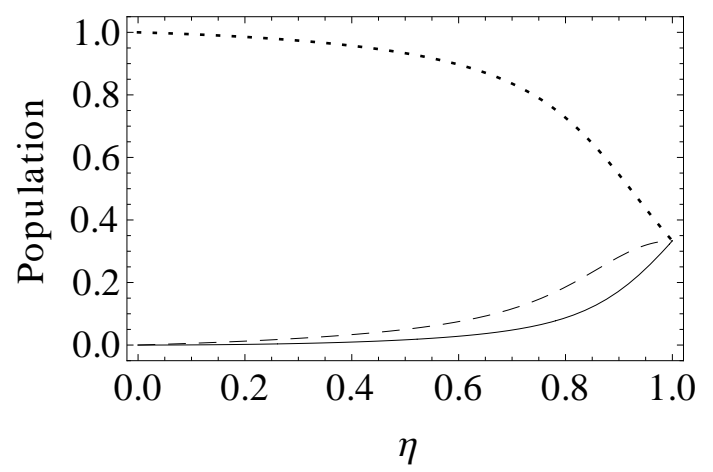

Figure 1. The steady-state dependence of the scaled populations $\left\langle S_{11}\right\rangle / N$ (solid line), $\left\langle S_{22}\right\rangle / N$ (dashed line) and $\left\langle S_{33}\right\rangle / N$ (dotted line) as a function of $\eta \equiv \eta_{1}=\eta_{2}$. Here $N=20$.

$\eta_{1}=\eta_{2} \equiv \eta=\bar{n} /[1+\bar{n}]$. The mean value of the inversion operator $\left(S_{z}=S_{11}-S_{33}\right)$, can be evaluated using the expression:

$$
\left\langle S_{z}^{k}\right\rangle_{s}=(-1)^{k} Z^{-1} \frac{\partial^{k}}{\partial \xi^{k}} Z(\xi), \quad\{k=1,2, \cdots\}
$$

Hence,

$$
\begin{aligned}
\left\langle S_{z}\right\rangle_{s} & =-\frac{N+\eta^{N+1}+2 \eta^{N+2}-(3+N) \eta^{3+2 N}}{\left(1-\eta^{N+1}\right)\left(1-\eta^{N+2}\right)} \\
& +\frac{\eta(1+3 \eta)}{1-\eta^{2}} .
\end{aligned}
$$

We consider the following limiting cases of Eq. (9):

i) a system in a weak incoherent reservoir $(\eta \rightarrow 0)$, has $\left\langle S_{z}\right\rangle_{s}=-N$;

ii) a fixed $\eta<1$ and a large atomic sample, $N \gg 1$, such that $\left\{\left(\eta_{1} \eta_{2}\right)^{N}, \eta_{1(2)}^{N}\right\} \rightarrow 0$, gives

$$
\left\langle S_{z}\right\rangle_{s}=-N+\frac{\eta(1+3 \eta)}{1-\eta^{2}}
$$

and

iii) a strong heat bath $(\eta \rightarrow 1)$ leads to saturation, i.e.

$$
\lim _{\eta \rightarrow 1}\left\langle S_{z}\right\rangle_{s} / N=0
$$

Concluding this part, we emphasize that a weak incoherent reservoir leaves a large sample of ladder emitters in their ground state, whereas equal distributions of populations among the states is achieved on applying a strong incoherent field. The coherence terms, that appear due to the quantum interference in the master equation do not affect the steady-state behaviors of radiators on the bare atomic states. It can be realized here that the steady-state inversion of atoms cannot be achieved in such a process, i.e., $\left\langle S_{11}\right\rangle_{s} \leq\left\langle S_{22}\right\rangle_{s} \leq\left\langle S_{33}\right\rangle_{s}$ or $\left\langle S_{z}\right\rangle_{s} \leq 0$ (see Fig. 1). The above conclusions also apply for $V$-type emitters interacting with a thermal reservoir [19]. 


\section{First- and second-order correlation functions}

The degree of second-order coherence of collective fluorescent light emitted from the excited atomic levels can be defined as [20, 21, 22],

$$
g^{(2)}(\vec{r}, t)=\frac{G^{(2)}(\vec{r}, t)}{\left[G^{(1)}(\vec{r}, t)\right]^{2}}
$$

with

$$
\begin{aligned}
& G^{(1)}(\vec{r}, t)=\left\langle\left[E^{-}(\vec{r}, t) E^{+}(\vec{r}, t)\right]\right\rangle, \\
& G^{(2)}(\vec{r}, t)=\left\langle:\left[E^{-}(\vec{r}, t) E^{+}(\vec{r}, t)\right]\left[E^{-}(\vec{r}, t) E^{+}(\vec{r}, t)\right]:\right\rangle,
\end{aligned}
$$

being the first- and the second-order correlation functions of the radiated EMF, respectively. Here $E^{-}(\vec{r}, t)$ and $E^{+}(\vec{r}, t)$ are the positive and negative frequency parts of the amplitude of the EMF operator $E$ at the space-point $\vec{r}$, and $:[\cdots]:$ means normal ordering. In the far-zone limit of experimental interest, i.e., $r=|\vec{r}| \gg\left\{\lambda_{12}, \lambda_{23}\right\}$, one can express the first- and the second- order correlation functions via the collective atomic operators. Taking then the long-time limit of Eq. (10) and making use of Eq. (2) the steady-state coherence properties of the generated EMF will be investigated in the next subsections.

\subsection{Photon statistics of distinguishable photons}

Let us consider that the atomic transitions $|1\rangle \rightarrow|2\rangle$ and $|2\rangle \rightarrow|3\rangle$ have dipole moments orthogonal to each other $\left(\vec{d}_{12} \perp \vec{d}_{23}\right)$. Then the emitted photons from the corresponding transitions can be distinguished by their polarizations and frequencies and can be detected by single-photon or two-photon detectors, respectively. For this case, the normalized second-order coherence function can be defined as follows:

$$
g_{i j}^{(2)}(0)=\frac{\left\langle J_{i}^{+} J_{j}^{+} J_{j} J_{i}\right\rangle}{\left\langle J_{i}^{+} J_{i}\right\rangle\left\langle J_{j}^{+} J_{j}\right\rangle}, \quad(i, j=1,2)
$$

where, for brevity we have set $J_{1}=S_{21}$ and $J_{2}=S_{32}$, and $\left\langle J_{i}^{+} J_{i}\right\rangle$ can be used to quantify the intensity of emitted light from the transition $i$. The quantity $g_{i j}^{(2)}(0)$ can be interpreted as a measure for the probability for detecting one photon emitted in transition $i$ and another photon emitted in transition $j$ simultaneously and its value determines the nature of the emitted photons. $g_{i j}^{(2)}(0)<1$ characterizes sub-Poissonian; $g_{i j}^{(2)}(0)>1$, super-Poissonian; and $g_{i j}^{(2)}(0)=1$, Poissonian photon statistics of the emitted EMF. Anti-correlation or correlation of the emitted light occurs when $g_{i j}^{(2)}(0)$ is smaller or larger than unity respectively. To evaluate these atomic correlation functions, we can use the $\mathrm{SU}(3)$ eigenstate properties of the bare state atomic operators [2, 4, 5, 7, 8] and the exact steady-state solution, i.e. the Eq. (2).

Firstly, we evaluate the fluorescent steady-state intensities of light emitted on $|1\rangle \rightarrow|2\rangle$ and $|2\rangle \rightarrow|3\rangle$, with help of the following relations obtained from the master equation (1):

$$
G_{1}^{(1)}(0) \propto\left\langle S_{12} S_{21}\right\rangle_{s}
$$




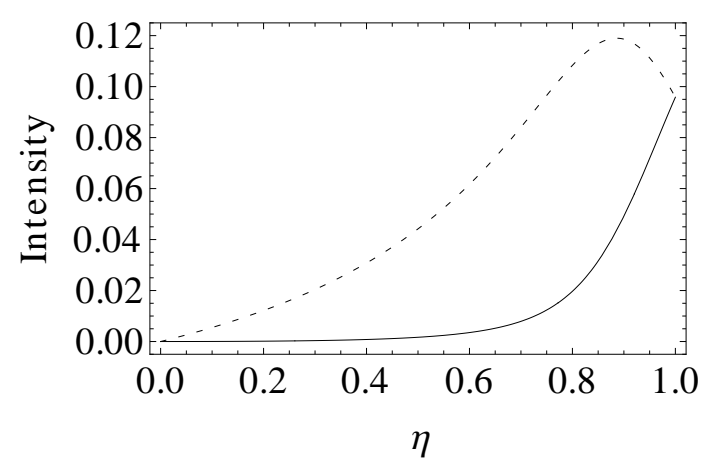

Figure 2. The steady-state dependence of the scaled intensities $G_{1}^{(1)}(0) / N^{2}$ (solid line) and $G_{2}^{(1)}(0) / N^{2}$ (dashed line) as a function of $\eta \equiv \eta_{1}=\eta_{2}$. Here $N=20$.

$$
\begin{aligned}
& =\frac{\eta_{1}}{1-\eta_{1}}\left[N-\left\langle S_{33}\right\rangle_{s}-2\left\langle S_{11}\right\rangle_{s}\right], \\
G_{2}^{(1)}(0) & \propto\left\langle S_{23} S_{32}\right\rangle_{s} \\
& =\frac{\eta_{2}}{1-\eta_{2}}\left[\left\langle S_{11}\right\rangle_{s}+2\left\langle S_{33}\right\rangle_{s}-N\right] .
\end{aligned}
$$

Particularly, Eqs. (12) and (13) were obtained from the steady-state form of the corresponding equations for $\left\langle S_{11}\right\rangle$ and $\left\langle S_{33}\right\rangle$ using also the commutation relations $\left[S_{12}, S_{21}\right]=S_{11}-S_{22}$ and $\left[S_{23}, S_{32}\right]=S_{22}-S_{33}$ as well as the relation $\left\langle S_{11}\right\rangle+\left\langle S_{22}\right\rangle+$ $\left\langle S_{33}\right\rangle=N$. Consider the following limiting cases for $G_{1}^{(1)}(0)$ and $G_{2}^{(1)}(0)$ :

i) if a weak incoherent field is applied to the sample, i.e., $\left\{\eta_{1}, \eta_{2}\right\} \rightarrow 0$, gives $G_{1}^{(1)}(0)$ $=G_{2}^{(1)}(0)=0$

ii) large samples, $N \gg 1$, with fixed $\left\{\eta_{1}, \eta_{2}\right\}<1$, have

$$
\begin{aligned}
& G_{1}^{(1)}(0)=\frac{\eta_{1}}{1-\eta_{1}}\left[\frac{\eta_{2}}{1-\eta_{2}}-\frac{\eta_{1} \eta_{2}}{1-\eta_{1} \eta_{2}}\right], \\
& G_{2}^{(1)}(0)=\frac{\eta_{2}}{1-\eta_{2}}\left[N-\frac{2 \eta_{2}}{1-\eta_{2}}-\frac{\eta_{1} \eta_{2}}{1-\eta_{1} \eta_{2}}\right],
\end{aligned}
$$

and

iii) in the strong field limit $\left\{\eta_{1}, \eta_{2}\right\} \rightarrow 1$ and fixed $N$, we find that,

$$
G_{1}^{(1)}(0)=G_{2}^{(1)}(0)=\frac{N}{12}(3+N) .
$$

One can observe here that for larger atomic systems and moderate strengths of incoherent excitation the first-order correlation function $G_{1}^{(1)}(0)$ does not depend on $N$ while $G_{2}^{(1)}(0)$ increases linearly with $N$, i.e., $G_{2}^{(1)}(0) \approx \bar{n}_{2} N$. In the limit of intense incoherent pumping the radiated fluorescence intensities in both atomic transitions scale as $N^{2}$, similar to the superradiance phenomenon [1, 2]. Fig. (2) depicts these intensities as a function of the incoherent pumping strength. An interesting result here is that $G_{2}^{(1)}(0) / N^{2}$ shows a maximum for lower pumping intensities. From Eq. (13), $G_{2}^{(1)}(0)$ can be written as $\bar{n}_{2}\left[\left\langle S_{33}\right\rangle_{s}-\left\langle S_{22}\right\rangle_{s}\right]$. The value of $G_{2}^{(1)}(0)$ increases with the pumping 


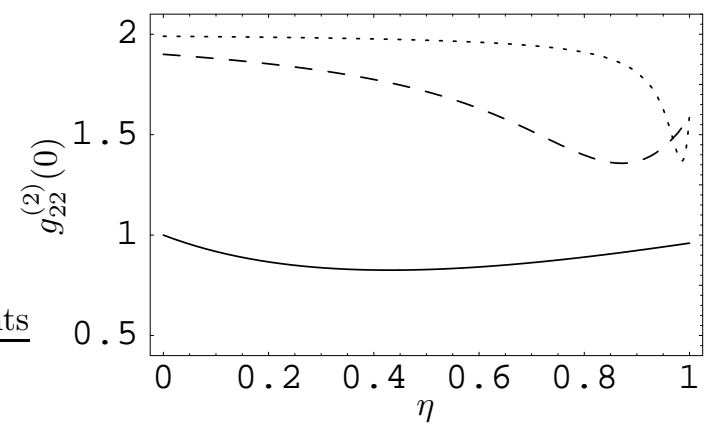

Figure 3. The steady-state dependence of the second-order coherence function $g_{22}^{(2)}(0)$ as function of $\eta \equiv \eta_{1}=\eta_{2}$. Here solid, dashed and dotted curves are for $N=2,20$, and 200 , respectively.

parameter $\eta$. After a certain value, the value of $G_{2}^{(1)}(0)$ decreases due to the rapidly decreasing nature of $\left[\left\langle S_{33}\right\rangle_{s}-\left\langle S_{22}\right\rangle_{s}\right]$, and hence exhibits a maximum due to amplified spontaneous emission by the external incoherent excitation.

We now shall investigate the coherence properties of the light emitted on $|2\rangle \rightarrow|3\rangle$ atomic transition. For $N=2$, the coherence factor $g_{22}^{(2)}(0)$ changes from unity (coherent light) to values less than one (i.e., it exhibits sub-Poissonian photon statistics, see Fig. 33). Hence, the emitted light possesses quantum features. For a moderately large atomic system, the fluorescent field generated on this particular atomic transition has partial coherent properties because $g_{22}^{(2)}(0)<2$. The light-statistics of a large sample behaves as follows. For a weak bath $(\eta<1)$ it is incoherent since $\lim _{N \rightarrow \infty} g_{22}^{(2)}(0)=2$, showing the super-Poissonian statistics of photons, while for an intense incoherent reservoir $(\eta=1)$, it is partially coherent, since

$$
\lim _{\eta \rightarrow 1} g_{22}^{(2)}(0)=\frac{8(N-1)(N+4)}{5 N(3+N)} \rightarrow \frac{8}{5}, \text { when } \mathrm{N} \gg 1 .
$$

It should be noted here that the minimum for the coherence factor $g_{22}^{(2)}(0)$, shown in Fig. (3), occurs near the value of $\eta$ for which $G_{2}^{(1)}(0)$ is maximum, leading to the emission of quasi-coherent light. Therefore, there occurs an enhancement of the multiparticle spontaneous emission corresponding to the maximum of $G_{2}^{(1)}(0)$ and quasi-coherent light emission corresponding to the minimum of $g_{22}^{(2)}(0)$ due to the surrounding incoherent reservoir and multi-level structure of the emitters in the system. The incoherent pumping scheme developed here for orthogonal dipoles can be useful in higher frequency domains due to absence of good coherent sources. As can be seen from our results, one can obtain quasi-coherent light via incoherent pumping. 


\subsection{Photon statistics of indistinguishable photons}

When decay interference effects are accounted, i.e., for near parallel dipoles $\left(\vec{d}_{12} \| \vec{d}_{23}\right)$, the second-order correlation function can be represented as follows:

$$
g^{(2)}(0)=\frac{\left\langle\left(J_{1}^{+}+J_{2}^{+}\right)^{2}\left(J_{1}+J_{2}\right)^{2}\right\rangle}{\left\langle\left(J_{1}^{+}+J_{2}^{+}\right)\left(J_{1}+J_{2}\right)\right\rangle^{2}} .
$$

It is emphasized here that due to the quantum decay interference the atomic transitions are indistinguishable. The correlation function, in this case, is detected by a two-photon detector. Eq. (17) contains off-diagonal terms that cannot be directly evaluated with the solution obtained in Eq. (2). However, we can represent the correlation functions entering Eq. (17) via those atomic correlations that can be evaluated with the steady-state solution in Eq. (2). Therefore, using the master equation, Eq. (11), we can show that:

$$
\begin{aligned}
G^{(1)}(0) & \propto\left\langle\left(J_{1}^{+}+J_{2}^{+}\right)\left(J_{1}+J_{2}\right)\right\rangle_{s}=-\frac{\eta}{1-\eta}\left\langle S_{z}\right\rangle_{s}, \\
G^{(2)}(0) \propto\left\langle\left(J_{1}^{+}+J_{2}^{+}\right)^{2}\left(J_{1}+J_{2}\right)^{2}\right\rangle_{s}= & \frac{\eta^{2}}{(1-\eta)^{2}}\left[\frac{1+\eta}{1-\eta}\left\langle S_{z}\right\rangle_{s}+2\left\langle S_{z}^{2}\right\rangle_{s}\right]
\end{aligned}
$$

and, thus, $g^{(2)}(0)$ can be written as,

$$
g^{(2)}(0)=\frac{[(1+\eta) /(1-\eta)]\left\langle S_{z}\right\rangle_{s}+2\left\langle S_{z}^{2}\right\rangle_{s}}{\left\langle S_{z}\right\rangle_{s}^{2}},
$$

with

$$
\begin{aligned}
\left\langle S_{z}^{2}\right\rangle_{s} & =\frac{a(\eta, N) \eta^{3+2 N}-b(\eta) \eta^{N+1}+c(\eta, N)}{(1-\eta)\left(1-\eta^{2}\right)\left(1-\eta^{N+1}\right)\left(1-\eta^{N+2}\right)} \\
& +\frac{2 \eta(1+3 \eta)}{1-\eta^{2}}\left\langle S_{z}\right\rangle_{s} .
\end{aligned}
$$

Here,

$$
\begin{aligned}
a(\eta, N) & =(3+N)^{2}-(2+N)(4+N) \eta+N(6+N) \eta^{3} \\
& -(1+N)(5+N) \eta^{2}, \\
b(\eta) \quad & =1+4 \eta-8 \eta^{3}-5 \eta^{4}, \\
c(\eta, N) & =N^{2}-\left(N^{2}-1\right) \eta-\left(N^{2}-4\right) \eta^{2}+\left(N^{2}-9\right) \eta^{3},
\end{aligned}
$$

and can be obtained from Eq. (8). Setting $N=1$ in Eqs. (9,20) we arrive at the corresponding expressions given in [23],

$$
\left\langle S_{z}\right\rangle_{s}=\frac{\eta^{2}-1}{1+\eta+\eta^{2}}, \quad\left\langle S_{z}^{2}\right\rangle_{s}=\frac{\eta^{2}+1}{1+\eta+\eta^{2}}
$$

The limiting cases for $G^{(1)}(0)$ in Eq. (18) are as follows:

i) for a weak incoherent field $(\eta \rightarrow 0)$ and fixed $N$, it can be shown that $G^{(1)}(0)=0$

ii) for a large sample $(N \gg 1)$ and a fixed $\eta<1$, we find that

$$
G^{(1)}(0)=-\frac{\eta}{1-\eta}\left[-N+\frac{\eta(1+3 \eta)}{1-\eta^{2}}\right] \approx \bar{n} N
$$




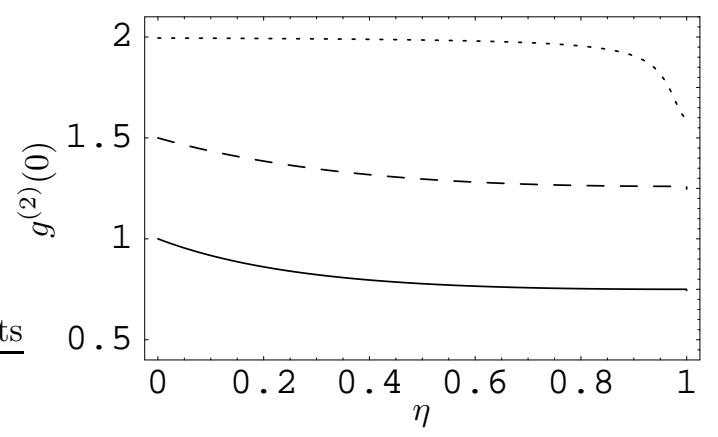

Figure 4. Steady-state variance of the second-order coherence function $g^{(2)}(0)$ as a function of the parameter $\eta$. Solid, dashed and dotted curves are for $\mathrm{N}=1,2$, and 200, respectively.

and

iii) for a strong incoherent field $(\eta \rightarrow 1)$ and fixed $N$, we get

$$
G^{(1)}(0)=\frac{N}{6}(3+N)
$$

Thus, in the limit of intense pumping and large atomic samples the radiated fluorescence intensity shows a quadratic dependence of $N$, whereas for moderate intensities it is a linear function of $N$. In this case, for atoms emitting indistinguishable photons, the scaled intensity does not show any maxima.

The values of the second-order correlation function for different limiting cases are as follows:

i) for $N=1$ and $\eta \neq 0$, we have,

$$
g^{(2)}(0)=1-\frac{\eta}{(1+\eta)^{2}} .
$$

The emitted light shows sub-Poissonian photon statistics. Increasing the number of atoms leads to partial coherence of the emitted electromagnetic field because $1<$ $g^{(2)}(0)<2$ (see Fig. 4)

ii) in the limit of a weak incoherent field $(\eta \rightarrow 0)$, we get

$$
g^{(2)}(0)=2-N^{-1}
$$

iii) in the limit of a strong incoherent bath $(\eta \rightarrow 1)$, we obtain

$$
g^{(2)}(0)=\frac{8 N^{2}+24 N-17}{5 N(3+N)} .
$$

and

iv) for fixed $\eta<1$ and large samples, $N \gg 1$, one obtains

$$
G^{(2)}(0) \approx 2(\bar{n} N)^{2}
$$

and, thus, $g^{(2)}(0)$ shows super-Poissonian photon statistics since in this case $\lim _{N \rightarrow \infty} g^{(2)}(0)=2$ [see Eqs. (2124)]. Partial coherence features occur for $\eta=1$ and $N \gg 1$ because $\lim _{N \rightarrow \infty} g^{(2)}(0)=8 / 5$ [see Eq. 23] . 
The second-order correlation function for a three-level system with near parallel dipoles behaves similar to that for a two-level sample [3]. This can be seen also by introducing new atomic operators, i.e., $S^{+}=\sqrt{2}\left(S_{23}+S_{12}\right), S^{-}=\sqrt{2}\left(S_{21}+S_{32}\right)$ and $S_{z}=S_{11}-S_{33}$ obeying the commutation relations for $\mathrm{su}(2)$ algebra: $\left[S^{+}, S^{-}\right]=2 S_{z}$ and $\left[S_{z}, S^{ \pm}\right]= \pm S^{ \pm}$. For equal decay rates, the master equation (1) can be represented via new operators as follows:

$$
\frac{d}{d t} \rho=-\frac{\gamma}{2}(1+\bar{n})\left[S^{+}, S^{-} \rho\right]-\frac{\gamma}{2} \bar{n}\left[S^{-}, S^{+} \rho\right]+H . c .,
$$

and looks like the master equation describing two-level atoms [3]. Hence, there is no amplified steady state spontaneous emission for such a system.

\section{Summary}

The interaction of an ensemble of ladder-type emitters with an environmental incoherent reservoir is investigated. The steady-state solution of the master equation and steadystate population distributions for the system are obtained and it is shown that collective effects force the system away from the Boltzmann-like thermodynamic equilibrium for systems with more than one atom. Particularly, the ground-state emitters obey the Bose-Einstein statistics. We analyzed the photon statistics of the emitted light under different conditions. The emitted EMF in the case of one or two-atom sample emitting distinguishable photons, or a single atom emitting indistinguishable photons exhibit quantum properties. In case of atoms, emitting distinguishable photons, for larger samples, amplified steady-state spontaneous emission of quasi-coherent light occurs. Therefore, the investigated model can be useful in higher frequency domains as a source of quasi-coherent light. Furthermore, the first- and second-order coherence functions do not exhibit any critical behaviors, i.e., discontinuities or abrupt changes proper to phase transition phenomena. Finally, the steady-state expectation values of any atomic variables of interest do not depend on spontaneous decay rates.

Rydberg atoms possessing almost equidistant energy levels and embedded inside a cavity with a low quality factor are suitable candidates to test some of the results described here [24]. With suitable cavity parameters one can avoid the difficulties connected with the condition, $\vec{d}_{12} \| \vec{d}_{23}$ [25].

\section{References}

[1] Dicke R H 1954 Phys. Rev. 93 99;

Shelepin L A Zh. Eksp. Teor. Fiz. 196854 1463;

Bonifacio R, Kim D M, Scully M O 1969 Phys. Rev. 187 441;

Gilmore R, Bowden C M, Narducci L M 1975 Phys. Rev. A 121019.

[2] Kiffner M, Macovei M, Evers J and Keitel C H 2010 Progress in Optics, edited by E. Wolf (North Holland, Amsterdam), Vol. 55, p. 85.

[3] Hassan S S, Hildred G P, Puri R R and Bullough R K 1982 J. Phys. B 152635. 
[4] Agarwal G S 1973 Physics Letters 45A 15;

Agarwal G S, Trivedi S S 1976 Optics Commun. 18 417;

Puri R R 1994 Phys. Rev. A 505309.

[5] Bogolubov N N Jr., Quang T and Shumovsky A S 1985 Physics Letters 112A 323;

Lawande S V, Jagatap B N 1988 Physics Letters 126A 329.

[6] Palma G M and Knight P L 1989 Phys. Rev. A 39, 1962;

Ficek Z 1990 Phys. Rev. A 42 611;

Knight P L and Quang T 1990 Phys. Rev. A 416255.

[7] Macovei M, Evers J and Keitel C H 2004 Europhysics Letters 68391.

[8] Macovei M, Evers J and Keitel C H 2005 Phys. Rev. A 72063809.

[9] Arnesen M C, Bose S and Vedral V 2001 Phys. Rev. Lett. 87017901.

[10] Basharov A M 2002 JETP Lett. 75 123;

Benatti F, Floreanini R, Piani M 2003 Phys. Rev. Lett. 91070402.

[11] Plenio M B and Huelga S F 2002 Phys. Rev. Lett. 88197901.

[12] Braun D 2002 Phys. Rev. Lett. 89277901.

[13] Galve F, Pachon L A, Zueco D 2010 Phys. Rev. Lett. 105 180501; Vedral V 2010 Nature 468, 769.

[14] Zou C-L, Zou X-B, Sun F-W, Han Z-F, Guo G-C 2011 Phys. Rev. A 84032317.

[15] Zampetaki A V and Diakonos F K 2012 Phys. Rev. Lett. 109090402.

[16] Allen L and Eberly J H, "Optical Resonance and Two-Level Atoms" (Dover, New York, 1975).

[17] Lawande S V, Jagatap B N 1989 Phys. Rev. A 39683.

[18] Martinez M A G, Herczfeld P R, Samuels C, Narducci L M, Keitel C H 1997 Phys. Rev. A 55, 4483;

Paspalakis E and Knight P L 1998 Phys. Rev. Lett. 81 293;

Keitel C H 1999 Phys. Rev. Lett. 83 1307;

Zhou P, Swain S and You L 2001 Phys. Rev. A 63 033818;

Ficek Z and Swain S 2002 J. Mod. Opt. 493.

[19] Agarwal G S and Menon S 2001 Phys. Rev. A 63023818.

[20] Glauber R J 1963 Phys. Rev. 130 2529;

Glauber R J 1963 Phys. Rev. 1312766.

[21] Loudon R 1983 The Quantum Theory of Light (Clarendon, Oxford).

[22] Walls D F and Milburn G J 1995 Quantum Optics (Springer - Verlag).

[23] Narducci L M, Bowden C M, Bluemel V, Carrazana G P 1975 Phys. Rev. A 11280.

[24] Beterov I M and Lerner P B 1989 Sov. Usp. Fiz. Nauk. 159665.

[25] Patnaik A K, Agarwal G S 1999 Phys. Rev. A 59 3015;

Zhou P, Swain S 2000 Opt. Comm. 179267. 